\section{A evolução regulatória e os desafios na perspectiva dos laboratórios públicos produtores de vacinas no Brasil}

\author{
Regulatory evolution and challenges from the \\ perspective of public laboratiories vaccine \\ producers in Brazil
}

\section{La evolución regulatoria y los desafíos desde la perspectiva de los laboratorios públicos productores de vacunas en Brasil}

\section{Resumo}

A regulamentação para produtos biológicos vem evoluindo rapidamente ao longo dos últimos anos, seja motivada por questões de qualidade com impacto na vida das pessoas, seja pelo advento de novas tecnologias. As mudanças nas regulamentações que ditam como um produto deve ser registrado, produzido e monitorado são constantes. A responsabilidade de reguladores e fabricantes na garantia da qualidade, segurança e eficácia das vacinas torna-se ainda mais crítica, uma vez que essas substâncias são utilizadas, em sua maioria, em crianças e em pacientes saudáveis. Diante desse cenário, fabricantes precisam criar estratégias para manter seus produtos e instalações adequadas e um sistema da qualidade atualizado e operante. Por outro lado, as agências reguladoras têm o papel de garantir que os produtos que estão em uso atendam aos critérios estabelecidos, sem comprometer o fornecimento de medicamentos para a população.

Vacinas; Autoridades de Saúde; Controle Social Formal
Monique Collaço de Moraes Stávale 1

Maria da Luz Fernandes Leal 1

Marcos da Silva Freire 1

doi: 10.1590/0102-311X00202219

\section{Correspondência}

M. C. M. Stávale

Instituto de Tecnologia em Imunobiológicos, Fundação Oswaldo Cruz.

Av. Brasil 4365, Rio de Janeiro, RJ 21040-900, Brasil. monique.moraes@bio.fiocruz.br

1 Instituto de Tecnologia em Imunobiológicos, Fundação Oswaldo Cruz, Rio de Janeiro, Brasil. 


\section{Introdução}

Os benefícios diretos e indiretos obtidos por meio da imunização são indiscutíveis. Seja pela considerável redução da mortalidade, seja pela diminuição do número de hospitalizações decorrentes de doenças preveníveis, as vacinas vêm historicamente desempenhando um papel ímpar na saúde pública, confirmando que prevenir é melhor do que tratar 1 .

Os primeiros registros de uso de vacinas com a introdução de versões atenuadas de vírus no corpo das pessoas estão relacionados ao combate à varíola no século $\mathrm{X}$, na China, onde as cascas de feridas provocadas pela doença eram trituradas, e o pó era assoprado no rosto das pessoas 2.

No entanto, foi em 1798 que o termo "vacina" surgiu pela primeira vez, por meio de uma experiência do médico e cientista inglês Edward Jenner. Ele ouviu relatos de que trabalhadores da zona rural não contraíam varíola, pois já haviam tido a varíola bovina, de menor impacto no corpo humano ${ }^{3}$. Em 1881, quando o cientista francês Louis Pasteur começou a desenvolver a segunda geração de vacinas, voltadas a combater a cólera aviária e o carbúnculo, ele sugeriu o termo para batizar sua recém-criada substância, em homenagem a Jenner ${ }^{4}$. A partir desse momento, as vacinas começaram a ser produzidas em massa e se tornaram essenciais para o combate de doenças. Com o aumento do volume da produção e a consequente ampliação do uso das vacinas ao redor do mundo, tornaram-se mais perceptíveis os problemas e eventos adversos causados por questões de qualidade e ausência de evidências clínicas das vacinas utilizadas.

A percepção da importância de uma avaliação independente dos medicamentos antes de entrar no mercado foi alcançada em diferentes momentos e regiões do mundo. Em muitos casos, a avaliação foi motivada por tragédias, como, por exemplo, o caso do cavalo chamado Jim, em 1901. Na ocasião, grande parte do soro coletado do cavalo foi dado a crianças com difteria. Com isso, 13 crianças contraíram tétano, a partir do sangue de Jim, e morreram. Foi verificado que nenhum teste de controle foi realizado nos lotes de soro. Esse incidente introduziu o conceito de adulteração na regulação de produtos biológicos, e o Congresso dos Estados Unidos promulgou a Lei de Controle de Biologia de 1902 5. Essa lei traçou um novo curso na política federal de saúde pública. Ela sustentou uma nova geração de leis posteriores voltadas à proteção do consumidor relativa a alimentos, medicamentos, cosméticos e outros produtos. A partir desse momento, vacinas, soros, toxinas e medicamentos biológicos começaram a ser regulados pelo Governo Federal do Estados Unidos, para controlar o comércio e assegurar que os produtos fossem seguros, puros e rotulados corretamente. Isso exigiu que os produtores obtivessem uma licença anual para a fabricação e venda de vacinas, soro e antitoxinas 6 .

As fábricas precisavam passar por inspeções, e as licenças podiam ser revogadas ou suspensas quando necessário. A produção teve que ser supervisionada por um cientista qualificado. Os produtos precisavam ser rotulados com o nome, a data de validade, o endereço e o número da licença do fabricante 6.

O Congresso também criou uma agência para implementar a lei: o Centro de Avaliação e Pesquisa Biológica da Administração de Alimentos e Medicamentos dos Estados Unidos (FDA, sigla em inglês).

Mesmo após as medidas acima implementadas, em 1955, as vacinas viriam a passar por mais um triste episódio, o "incidente Cutter". Nessa tragédia, a produção da vacina de poliomielite inativada em instalações industriais operadas pela empresa Cutter, levou a vacinação de 200 mil pessoas com cepa virulenta da poliomielite. Podemos afirmar que esse foi uns dos mais graves episódios da história da vacinação, já que 70 mil pessoas adoeceram, duzentas ficaram permanentemente paralíticas e dez morreram. O incidente Cutter deixou um legado para a regulamentação sanitária, pois efetivou a regulação federal das vacinas, que hoje gozam de uma avaliação de segurança inigualável quando comparadas a qualquer outro produto médico 6 .

A decisão judicial de que a Cutter era responsável pelo pagamento de indenização abriu precedente para uma série de litígios. Como resultado, as vacinas estavam entre os primeiros produtos médicos quase eliminados por processos judiciais. Diante desse cenário, em 1986, o Programa Nacional de Compensação de Lesões por Vacinas foi introduzido para proteger os fabricantes de vacinas de processos judiciais. Ainda assim, muitas empresas optaram por sair desse campo 7 .

A partir de 1962, os fabricantes passaram a apresentar "evidência substancial” sobre eficácia e segurança dos produtos a serem comercializados. Os demais países, tivessem ou não iniciado con- 
troles de registro de produtos anteriormente, nas décadas de 1960 e 1970, viram um rápido aumento nas leis, nos regulamentos e nas diretrizes para relatar e avaliar os dados sobre segurança, qualidade e eficácia de novos medicamentos 8 .

A indústria estava se tornando mais internacional e buscando novos mercados globais, no entanto a divergência de requisitos técnicos de país para país era tal que a indústria considerou necessário duplicar muitos procedimentos e testes, demorados e caros, para comercializar novos produtos internacionalmente. A Europa avançou no desenvolvimento de um mercado único para os produtos farmacêuticos, e o sucesso alcançado nesse continente demonstrou que a harmonização era viável 9 .

Assim, foram iniciadas discussões entre Europa, Japão e Estados Unidos sobre possibilidades de harmonização. Os planos de ação começaram a se materializar na Conferência de Autoridades Reguladoras de Medicamentos da Organização Mundial da Saúde (ICDRA, sigla em inglês), em Paris, em 1989. Pouco tempo depois, as autoridades se aproximaram da Federação Internacional de Fabricantes e Associações Farmacêuticas (IFPMA, sigla em inglês) para discutir uma iniciativa conjunta do setor regulador sobre harmonização internacional e, nesse contexto, foi criado o Conselho Internacional para Harmonização de Requisitos Técnicos para Produtos Farmacêuticos para Uso Humano (ICH, sigla em inglês) 10 .

O ICH reúne as autoridades reguladoras e a indústria farmacêutica para discutir os aspectos científicos e técnicos do registro de medicamentos. Desde a sua criação, o ICH vem evoluindo gradualmente, e sua missão é alcançar uma maior harmonização mundial para garantir que medicamentos seguros, eficazes e de alta qualidade sejam desenvolvidos e registrados da maneira mais eficiente em termos de recursos 10 .

No Brasil, o controle de drogas, alimentos, vacinas e outros produtos voltados para saúde era feito de forma inconsistente, e as análises eram realizadas pelo Laboratório Central de Controle de Drogas, Medicamentos e Alimentos (LCCDMA) 11.

Em 23 de setembro de 1976, finalmente o Brasil passou a dispor de um marco sanitário legal para medicamentos, por meio da criação da Lei no 6.36012 e do Decreto no 79.09413 publicado em 5 de janeiro de 1977. A partir da Lei no 6.360, os estabelecimentos responsáveis por fabricação, importação, armazenamento, distribuição, entre outras funções, deveriam ser autorizados pelo Ministério da Saúde para o início da execução dessas atividades.

A Agência Nacional de Vigilância Sanitária (Anvisa) foi criada somente em 26 de janeiro de 1999, por meio da Lei no 9.78214 , com a finalidade de promover a proteção da saúde da população por intermédio do controle sanitário da produção e do consumo de produtos e serviços submetidos à vigilância sanitária, inclusive dos ambientes, dos processos, dos insumos e das tecnologias a eles relacionados.

A Anvisa foi então estruturada para atender às necessidades da sociedade e do mercado, e iniciou o processo de construção do arcabouço regulatório sanitário hoje composto de centenas de documentos. O processo de qualificação do mercado farmacêutico iniciado com a criação da Anvisa passou então a ser constante e, a partir desse momento, para obterem o registro sanitário e consequentemente a autorização para distribuição e comercialização de medicamentos no país, os fabricantes precisam cumprir uma etapa de legalização que compreende basicamente as autorizações e licenças de funcionamento, o registro sanitário onde serão avaliados os dados de produção, qualidade, segurança e eficácia, além de uma etapa de inspeção ao local de fabricação, momento em que serão avaliadas as condições de boas práticas de fabricação (BPF) 15.

Com exceção da etapa de legalização, em que não há mudanças significativas ao longo do tempo, os demais elementos que compõem as etapas que um fabricante deve cumprir antes de disponibilizar seu produto para uso passaram por significativas mudanças desde a criação da Anvisa e publicação do primeiro marco regulatório até a atualidade. 


\section{A regulamentação sanitária para registro de produtos biológicos}

No que se refere à regulamentação sobre informações a serem apresentadas para solicitação de registro, apesar de a Anvisa ter sido criada em 1999, o primeiro marco regulatório para produtos biológicos foi publicado em 1994, por meio da Portaria no 107 e, na ocasião, o Instituto Nacional de Controle de Qualidade em Saúde, Fundação Oswaldo Cruz (INCQS/Fiocruz) tornou-se um dos braços da Secretaria de Vigilância Sanitária, Ministério da Saúde, quando da implantação da Política Nacional de Saúde 16.

Em 2002, produtos biológicos ganham a primeira regulamentação emitida pela Anvisa: a RDC no 80/200217. Essa Resolução distinguiu os produtos biológicos entre aquele que "contém molécula com atividade biológica conhecida" e aquele que "contém molécula com atividade biológica nova e tem proteção patentária”, sendo o segundo atribuído aos produtos biológicos novos no país. A RDC no 80/2002 exigia que fossem apresentadas informações de produção e controle de qualidade simples e, excepcionalmente, o fabricante poderia solicitar à Agência a substituição dos estudos (fases II e III) por outros documentos comprobatórios de segurança e eficácia clínica.

Para solucionar o passivo de produtos que estavam no mercado, a Anvisa separou os produtos biológicos em três grupos conforme o período de solicitação do registro. Sendo assim, os produtos que estavam em análise na ocasião da publicação da RDC teriam um ano para se adequarem; os já registrados receberiam um prazo de dois anos para adequação, e as novas solicitações deveriam atender ao novo formato proposto imediatamente na solicitação do registro 17 .

De modo relativamente rápido, três anos após a publicação da primeira regulamentação, em 2005, um novo marco para registro de medicamentos biológicos foi estabelecido pela RDC no 315/2005 18. Essa RDC apresentava-se mais rigorosa que sua antecessora, e informações sobre a descrição da etapa do processo de fabricação, validação da cadeia de transporte e estudos clínicos de não inferioridade para os medicamentos biológicos não considerados novos passaram a ser mandatórios. Apesar de mais rigorosa, a RDC no 315/2005 não orientava os fabricantes quanto às diferentes categorias de produtos biológicos.

Em 2010, a Anvisa publicou a RDC no 55/2010 19 e instituiu um novo marco regulatório, além de novas definições para produtos biológicos e produtos biológicos novos: " $X V$ - Produto Biológico: é o medicamento biológico não novo ou conhecido que contém molécula com atividade biológica conhecida, já registrado no Brasil e que tenha passado por todas as etapas de fabricação (formulação, envase, liofilização, rotulagem, embalagem, armazenamento, controle de qualidade e liberação do lote de produto biológico para uso). (...) XX - Produto Biológico Novo: é o medicamento biológico que contém molécula com atividade biológica conhecida, ainda não registrado no Brasil e que tenha passado por todas as etapas de fabricação (formulação, envase, liofilização, rotulagem, embalagem, armazenamento, controle de qualidade e liberação do lote de medicamento biológico novo para uso)".

Essa resolução trouxe também necessidade de estudos de imunogenicidade, plano de farmacovigilância e de minimização de riscos. Outra inovação apresentada pela RDC em relação às anteriores foram as recomendações específicas aos diferentes tipos de medicamentos biológicos. Assim, vacinas, hemoderivados e produtos de origem biotecnológicas receberam requerimentos diferenciados. Podemos dizer que a RDC no 55/2010 foi um avanço em termos de regulamentação e colocou o Brasil alinhado a práticas regulatórias internacionais.

Outro avanço na regulamentação de medicamentos biológicos foi a RDC no 49/201120, que definiu as alterações pós-registro. Até então, os tais requerimentos eram tratados dentro da mesma regulamentação destinada a registro de produtos biológicos. A RDC no 49/2011 categorizou as alterações em três níveis conforme a complexidade: as alterações de nível 1 não precisam de anuência prévia da Anvisa para implementação, já as de níveis 2 e 3 somente poderão ser implementadas após a aprovação da Agência. 


\section{A regulamentação sanitária para boas práticas de fabricação}

Somada à eficácia, à segurança e ao acesso, a qualidade é um dos quatro pilares estabelecidos pela OMS que sustentam a produção de medicamentos, sendo indispensável a qualquer produto voltado para a saúde. Um importante marco histórico para o estabelecimento de requisitos das BPFs foi a 28 a Assembleia Mundial de Saúde, promovida pela OMS, em maio de 1975. Naquele fórum, foi aprovado o Guia de Boas Práticas de Fabricação para a Indústria Farmacêutica, o qual deu base à Portaria SVS/

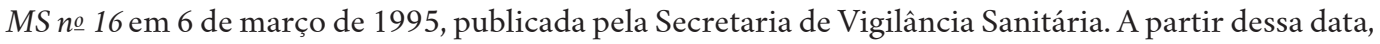
a indústria farmacêutica passaria a contar com uma versão nacional dos requisitos de BPFs 21,22.

A criação da Anvisa em 1999 trouxe ao setor uma nova dinâmica de regulação, em que a Agência passaria a atuar nos diversos serviços e produtos voltados para a saúde por meio de um ambiente regulatório definido associado a fiscalização 15 .

Dessa forma, a Agência publica a primeira regulação destinada às BPFs de medicamentos. Trata-se da $R D C$ no 134/2001 23, que considerou as recomendações da OMS sobre certificação de qualidade de produtos farmacêuticos, padronizou as ações de vigilância sanitária e trouxe claramente o caráter fiscalizador da Agência para fins de verificação das BPFs, por meio do estabelecimento de um roteiro de inspeção. A maior parte dos itens propostos pela RDC no 134/2001 deveria ser implementada imediatamente, com algumas exceções, em que os fabricantes teriam um prazo de 18 meses. Entre as exceções que teriam um prazo maior para implementação, estavam a validação de envase asséptico de produtos estéreis, a instalação do liofilizador na área de envase asséptico e a validação de metodologia analítica.

Em 2003, a Anvisa revogou a RDC no 134/2001 com a publicação da $R D C$ no 210/2003 24. Como novidade, a Resolução instituiu a classificação e os critérios de avaliação dos itens descritos no roteiro de inspeção para fabricantes de medicamentos com base no potencial de risco na qualidade e segurança inerentes ao processo produtivo. Além disso, confirmou a obrigatoriedade da validação de métodos analíticos 4 .

A $R D C$ no 17/201025 substituiu a RDC no 210/2003 e trouxe uma série de mudanças de impacto para a indústria farmacêutica, principalmente para os fabricantes de produtos estéreis. A RDC no 17/2010 estabeleceu a necessidade de validação de sistemas computadorizados, e de investigação de resultados fora das especificações durante o controle de qualidade, foram incluídos ainda itens para água para uso farmacêutico, que até aquele momento não possuía uma regulamentação apropriada.

Além disso, duas mudanças críticas levaram à paralisação de laboratórios públicos, com consequências para o fornecimento de vacinas ao Programa Nacional de Imunizações (PNI). Uma delas foi a restrição para o compartilhamento de áreas e outra foi quanto ao grau das áreas limpas utilizadas para a fabricação dos medicamentos. Os fabricantes passaram de um cenário em que o requerido era grau A circundado por C para um cenário de grau A circundado por B. Essa adequação exigiu que fabricantes paralisassem suas instalações para obras e exigiu também a implantação de novos sistemas de ar condicionado, tendo alguns fabricantes públicos ficado por longo período com suas instalações inoperantes, comprometendo o fornecimento de vacinas para o PNI.

Em agosto de 2019, a Anvisa publicou a RDC no 301/2019 26. Essa versão das BPFs apresenta um formato relativamente diferente das suas antecessoras, uma vez que somente os aspectos comuns ao sistema de qualidade farmacêutico foram publicados no formato de RDC. As regras específicas, como por exemplo produtos estéreis, biológicos e validação de sistemas, foram publicadas no formato de Instruções Normativas. O novo formato tem como objetivo dar mais flexibilidade e celeridade à atualização dos requisitos BPFs, uma vez que o fluxo regulatório para mudança de uma Instrução Normativa é mais simples do que o fluxo de uma RDC. Enquanto a RDC anterior se baseava no guia da OMS de 2003, a norma atual segue o Pharmaceutical Inspection Co-operation Scheme (PIC/S), já que a Agência está em processo de adesão a esse sistema de cooperação entre agências regulatórias no campo das BPFs. No que se refere aos aspectos técnicos, a nova regulamentação ampliou o rigor aos elementos que compõem o sistema da qualidade farmacêutico e a necessidade do envolvimento e comprometimento da alta gestão, refletindo a sua proximidade com os guias internacionais. Além disso, é possível observar um maior detalhamento dos requisitos, o que traz facilidade às empresas na implementação.

O compartilhamento de áreas volta a ser permitido, desde que a empresa, por meio de ferramentas de análise de riscos, comprove validação de limpeza, que a remoção dos resíduos da produção anterior foi realizada de forma efetiva e que não oferece risco de contaminação cruzada e análises toxicológicas 
dos produtos ali processados. Se, por um lado, a norma se mostra mais flexível nos requisitos aplicáveis a instalações e processos, por outro, instrumentos de gestão receberam maior peso e responsabilização, trazendo para as companhias a necessidade de estabelecimento de um processo robusto de gerenciamento de riscos 26 .

A RDC no 301/2019 possui uma maior abrangência, uma vez que em sua Instrução Normativa no 4527 , a Agência traz diretrizes para boas práticas de fabricação para os medicamentos experimentais.

Em relação à transitoriedade, a RDC no 301/2019 entrou em vigor 45 dias após sua publicação, com exceção de alguns itens, onde foi concedido um prazo maior para implementação, como, por exemplo, o estabelecimento de um processo de gerenciamento de riscos da qualidade que inclua avaliação toxicológica e de potência para avaliar e controlar os riscos de contaminação cruzada apresentados pelos produtos fabricados. Nesse caso, a implementação poderá ser realizada de forma gradual, iniciandose pela estruturação e capacitação, com o prazo de seis meses, até o total cumprimento, cobrindo 100\% do portfólio, tendo a empresa terá 48 meses para implementar o requisito 26.

\section{A regulamentação sanitária para pesquisa clínica}

Assim como as BPFs e as informações sobre produção e controle de qualidade de um medicamento, as informações de segurança e eficácia são partes essenciais do pacote regulatório que será avaliado pela Agência para a concessão do registro sanitário.

No entanto, no caso da pesquisa clínica, existem duas grandes avaliações: a ética, que irá considerar se o protocolo proposto está em conformidade com a regulamentação nacional e internacional para a condução de pesquisa em seres humanos salvaguardando a segurança e o bem-estar dos participantes da pesquisa, e uma técnica-regulatória, voltada para questões sanitárias.

Considerando que o tema do artigo está relacionado aos aspectos técnico-sanitários, o texto se concentrará na evolução dos requisitos solicitados pela Anvisa nas avaliações e aprovações da pesquisa clínica no país.

Assim como ocorreu para os demais elementos que formam o registro, a regulamentação sanitária para pesquisa clínica vem evoluindo gradativamente. Antes da criação da Anvisa, o documento que previa os requisitos sanitários para pesquisa no Brasil era a Portaria no 911 28, de 12 de novembro de 1998. Trata-se de um documento publicado pela Secretaria de Vigilância Sanitária, com redação simples, em que estavam listados 11 documentos que deveriam ser apresentados nos pedidos de autorização para realização de pesquisa clínica com fármacos, medicamentos, vacinas e testes diagnósticos novos. Apesar da superficialidade do documento, as questões éticas foram preservadas, uma vez que entre os requisitos listados estavam a necessidade de comprovação de que o Comitê de Ética em Pesquisa (CEP) da instituição onde seria realizada a pesquisa estava registrado e aprovado na Comissão Nacional de Ética em Pesquisa (CONEP) e do parecer consubstanciado do CEP da instituição onde seria realizada a pesquisa, aprovando o protocolo clínico e seu consentimento livre e esclarecido.

Após a sua criação, a primeira regulamentação de pesquisa clínica publicada pela Anvisa foi a $R D C$ no 219/200429, a qual estabeleceu o regulamento para elaboração de dossiê para a obtenção de Comunicado Especial (CE) para a realização de pesquisa clínica com medicamentos e produtos para a saúde. A $R D C$ no 219/2004 trouxe a necessidade de maior detalhamento dos documentos que deveriam ser apresentados na solicitação de pesquisa clínica, e colocou a possibilidade de a Agência realizar auditorias para verificação do grau de aderência às boas práticas clínicas e à legislação brasileira vigente.

Essa Resolução foi alvo de críticas ao estabelecer que, no caso de pesquisas multicêntricas, deveria ser solicitado um CE para cada centro participante, o que gerava demora para aprovação da pesquisa clínica 30 .

Em junho de 2008, a Anvisa publicou a RDC no 39/200831, passando a ser este o marco regulatório válido. A RDC no 39/2008 buscou colocar a regulamentação brasileira nos moldes internacionais, pois permitiu que estudos que tivessem sido analisados e aprovados em países com agências regulatórias de referência tivessem um processo de análise simplificada. Na ocasião foram consideradas as seguintes: FDA - Estados Unidos; Agência Europeia de Medicamentos (EMA, sigla em inglês) - União Europeia; Agência de Aconselhamento Farmacêutico e Médico (PMDA, sigla em inglês) - Japão; e Saúde Canadá - Canadá. 
A partir da $R D C$ no 39/2008, todos os estudos fases I, II, III e IV passaram a ter que apresentar comprovante de registro de pesquisa clínica na base de dados do Registro Brasileiro de Ensaios Clínicos (ReBEC) ou comprovante de submissão. Para casos anteriores à publicação da resolução, foram aceitos comprovantes de registro das pesquisas na Plataforma Internacional de Registro de Ensaios Clínicos (ICTRP, sigla em inglês) 32 .

Outro ponto positivo da $R D C$ no 39/2008 foi a avaliação simultânea para todos os centros de pesquisa, com emissão de um único CE por estudo, o que tornou o processo de aprovação do estudo relativamente mais rápido.

Atualmente o marco regulatório válido para pesquisa clínica é $R D C$ no 9/201532, publicada em 20 de fevereiro de 2015, a qual revogou a RDC no 39/2008 e trouxe o conceito do Dossiê de Desenvolvimento Clínico do Medicamento (DDCM) e Dossiê Específico de Ensaio Clínico. Enquanto o segundo concentra as informações para cada estudo, como, por exemplo, o protocolo de pesquisa e comprovante de registro na base de dados, o primeiro, DDCM, será composto do conjunto de informações e documentos sobre todas as etapas de desenvolvimento do produto até o momento da submissão. Confirmando seu compromisso com o desenvolvimento da pesquisa no país, e entendendo que os longos prazos de análise prejudicam a credibilidade nacional e internacional da Agência, com a publicação da $R D C$ no 9/2015, a Anvisa buscou estabelecer estratégias para dar celeridade às avaliações dos processos, sem prejuízo à qualidade técnica.

\section{Discussão}

A Anvisa, hoje reconhecidamente uma Agência forte, desempenha um importante papel no fortalecimento da indústria farmacêutica brasileira. Produtos registrados no Brasil são considerados seguros e com qualidade garantida. A evolução do processo de inspeção da Agência é visível. Partiu de uma avaliação baseada em itens de verificação (check list) em 2003, por meio da RDC no 134/2003, e alcançou, em 2019, uma inspeção baseada em riscos, em que o sistema da qualidade é avaliado de forma sistêmica.

Tal evolução foi necessária para o aprimoramento da indústria nacional, assim como para o alcance de padrões de qualidade atualmente exigidos, no entanto, o ônus foi o encerramento das atividades de diversos laboratórios e a descontinuação de importantes medicamentos. Nos laboratórios públicos o cenário não foi diferente, pois o aumento no grau das exigências, que em parte apontava para reformas, associadas a dificuldades orçamentárias e também a trâmites burocráticos, fez com que esses laboratórios parassem temporariamente e, em alguns casos, definitivamente com suas produções.

A Anvisa, mantendo a estratégia de convergência regulatória, tornou-se integrante do PIC/S e baseou seu novo marco regulatório, a RDC no 301/2019, no conjunto de requisitos que direciona as principais agências regulatórias internacionais.

A nova RDC está coerente com as discussões prévias à publicação promovida pela própria Agência com as partes interessadas. Dessa forma, confirmou-se o fortalecimento do conceito de sistema da qualidade farmacêutico, e não mais garantia de qualidade, consolidação das ferramentas de gerenciamento de riscos e sua abordagem ao longo do ciclo de vida do produto.

$\mathrm{O}$ fato de a nova RDC não trazer requisitos que levem a questões estruturais complexas como as anteriores não torna menos desafiantes as adequações que laboratórios públicos terão de implementar, pois desta vez a reforma necessária está ligada a cultura. Integridade de dados, gerenciamento de riscos e um sistema de gestão da qualidade forte são as novas exigências que demandarão dos fabricantes investimentos em capacitação dos seus colaboradores.

O compartilhamento de áreas voltou a ser permitido mediante avaliação de riscos e adequação aos processos de validação e análise toxicológica. Esse é um importante ponto, dado que no passado, com a publicação da $R D C$ no 17/2010, tal compartilhamento havia sido proibido, e indústrias farmacêuticas, mesmo tendo seus processos validados e qualificados, precisaram optar por quais produtos seriam mantidos e quais seriam retirados do portfólio. Na ocasião, a RDC no 17/2010 havia sido baseada no guia da OMS, que permitia o compartilhamento em casos especiais e, na sua tradução, a $R D C$ $n$ ํ. 17/2010 entendeu esses casos como calamidades públicas. O impacto desse ponto nos laboratórios públicos foi enorme. No Instituto de Tecnologia em Imunobiológicos - Bio-Manguinhos, Fundação 
Oswaldo Cruz, a opção foi interromper a produção da vacina polissacarídica menigocócia AC para a manuteção da vacina de febre amarela, uma vez que vacinas de vírus vivo atenuado e vacinas de subunidades ou inativadas não poderiam ser envasadas na mesma linha, mesmo que por campanha.

Apesar de o compartilhamento de áreas estar previsto na nova legislação, ainda estão sendo discutidos os critérios a serem adotados e, mais uma vez, será necessário investimento em formação de pessoal, como toxicologistas com visão de validação de limpeza, que serão essenciais para atuar nesse processo.

No que se refere à etapa de registro de produtos, assim como ocorreu para as BPFs, a resolução de registros de produtos biológicos também merece revisão. É importante lembrar que a regulamentação que dispõe sobre registro de produtos biológicos, RDC no 55/2010, foi publicada em 2010 e, considerando que estamos em 2020, temos dez anos de avanços no campo da biotecnologia, que podem não estar cobertos por uma regulamentação, gerando atraso no tempo de resposta da Agência.

Medicamentos obtidos a partir de animais transgênicos e de plataformas vegetais são exemplos de onde ainda não existem recomendações específicas. A falta ou inexistência de um documento regulatório com diretrizes para algumas tecnologias traz inseguranças ao fabricante, pois o dossiê de registro será analisado caso a caso.

Existem algumas iniciativas, ainda incipientes, de aproximação da Agência com as universidades para construção e atualização de requisitos regulatórios. O fortalecimento dessa parceria trará importantes ganhos no tempo de resposta da Agência a novas tecnologias e na construção de uma regulamentação baseada na ciência.

É importante destacar que a Agência atua baseada no princípio da precaução, ou seja, diante de uma nova situação ou tecnologia, o posicionamento será o mais conservador possível, garantindo, assim, a segurança da população e confirmando o seu papel de regulador sanitário.

Algumas alternativas vêm sendo adotadas pela Agência, para que esforços e tempos sejam concentrados em produtos de maior risco. Nesse sentido, em março de 2019, a Agência publicou a Consulta Pública no 633/2019, que estabelece os requisitos mínimos para o registro de produtos biológicos de menor complexidade.

A Anvisa também busca alinhamento com as práticas regulatórias mundiais para registro de produtos e, em novembro de 2016, com a entrada como membro do ICH, a Agência passou a compor 18 grupos de trabalho que discutem novos requisitos e a atualização dos já existentes nos temas cobertos pelo ICH.

Ao tornar-se membro, a Anvisa se comprometeu a implementar cinco guias em cinco anos e aproximadamente 60 guias em um prazo a ser definido, em um plano de ação, além daqueles que tiveram suas implementações imediatas.

Um dos guias a serem implementados em cinco anos é o Commom Technical Document (CTD), que trata do formato e do conteúdo sobre como a documentação de registro deve ser submetida para avaliação da Agência. Esse formato é adotado por muitos países, e o objetivo é que um único dossiê possa ser aplicado em diferentes agências regulatórias, evitando retrabalho dos fabricantes e consequentemente a disponibilização mais rápida de produtos promissores. Infelizmente, na prática, como existem particularidades locais, o objetivo de um documento único para registro ainda não foi alcançado. No entanto, sendo a Anvisa parte desse grupo, o Brasil acompanhará de perto essa evolução.

\section{Conclusão}

Podemos perceber que, enquanto no passado o avanço da regulamentação foi motivado por tragédias e questões de qualidade, na atualidade são as novas tecnologias e metodologias mais sensíveis que movem as agências reguladoras a publicarem novos requisitos. No entanto, ao tornarem públicas novas exigências, as agências devem fazer uma avaliação criteriosa se de fato o novo requisito agrega valor à qualidade, à segurança e à eficácia, para que não se torne uma barreira que irá paralisar diversos fabricantes, principalmente de países em desenvolvimento, e colocar em risco o suprimento de vacinas que comprovadamente já imunizaram populações inteiras.

Os laboratórios públicos vivenciam diariamente esse desafio. Se, por um lado, temos uma agência regulatória reconhecida, tornando-se internacional com a participação em fóruns que discutem o 
destino da regulamentação farmacêutica mundial, como por exemplo, o PIC/S e o ICH, por outro lado, esses mesmos laboratórios precisam lidar com as restrições aplicadas a órgãos públicos, onde os tempos requeridos para reformas e implementação de requisitos nem sempre acompanham as mudanças regulatórias. Pode-se perceber que a $R D C$ no 301/2019, recentemente publicada, traz algumas mudanças no cenário, visto que, no passado, as atualizações da norma geravam grandes impactos em obras e instalações. Dessa vez, um sistema da qualidade forte e o comprometimento da alta gestão passam a ser o ponto central da discussão. Essa mudança de abordagem, no entanto, não torna menores os desafios dos laboratórios públicos que, além da atualização do parque industrial, devem também investir em capacitação e modernização da gestão.

\section{Colaboradores}

M. C. M. Stávale colaborou na revisão bibliográfica e redação do artigo. M. L. F. Leal contribuiu na concepção e desenho do artigo, análise e interpretação dos dados e revisão do conteúdo do artigo. M. S. Freire colaborou na revisão crítica do conteúdo do artigo.

\section{Informações adicionais}

ORCID: Monique Collaço de Moraes Stávale (00000001-7051-8233); Maria da Luz Fernandes Leal (0000-0002-8886-8116); Marcos da Silva Freire (0000-0002-4723-8994).

\section{Agradecimentos}

Ao Luiz Antonio Camacho (Escola Nacional de Saúde Pública Sergio Arouca, Fundação Oswaldo Cruz) e ao Instituto de Tecnologia em Imunobiológicos (Bio-Manguinhos), Fundação Oswaldo Cruz.

\section{Referências}

1. Rappuoli R, Mandl CW, Black S, De Gregorio E. Vaccines for the twenty-first century society. Nat Rev Immunol 2011; 11:865-72.

2. Henderson DA, Moss B. Smallpox and vaccinia. In: Plotkin SA, Mortimer Jr. EA, editors. Vaccines. 3rd Ed. Philadelphia: WB Saunders; 1999. p. 74-97.

3. Smith KA. Edward Jenner and the small pox vaccine. Front Immunol 2011; 2:21.

4. Plotkin SA. History of vaccine development. Nova York: Springer; 2011.

5. Bren L. The road to the biotech revolution highlights of 100 years of biologics regulation https://www.fda.gov/files/about\%20fda/published/The-Road-to-the-Biotech-RevolutionHighlights-of-100-Years-of-Biologics-Regula tion.pdf (acessado em 19/Jul/2019).

6. Coleman TC. Early developments in the regulation of biologic. Food and Drug Law Journal 2016; 71:544-607.

7. Cook KM, Evans G. The national vaccine injury compensation program. Pediatrics 2011; 127 Suppl 1:S74-7.

8. Hooper CL. Pharmaceuticals: economics and regulation. https://www.econlib.org/library/ Enc/PharmaceuticalsEconomicsandRegula tion.html (acessado em 05/Jul/2019).

9. Vogel D. The globalization of pharmaceutical regulation. Governance 1998; 11:1-22.

10. International Council of Harmonization. About ICH: history. https://www.ich.org/ about/history.html (acessado em 05/Jul/2019).

11. Ponte CF. Vacinação, controle de qualidade e produção de vacinas no Brasil a partir de 1960. Hist Ciênc Saúde-Manguinhos 2003; 10 Suppl 2:619-53.

12. Brasil. Lei no 6.360, de 23 de setembro de 1976. Dispõe sobre a Vigilância sanitária a que ficam sujeitos os medicamentos, as drogas, os insumos farmacêuticos e correlatos, cosméticos, saneantes e outros produtos, e dá outras providências. Diário Oficial da União 1976; 24 set. 
13. Brasil. Decreto no 79.094 , de 5 de janeiro de 1977. Regulamenta a Lei no 6.360, de 23 de setembro de 1976, que submete a sistema de vigilância sanitária os medicamentos, insumos farmacêuticos, drogas, correlatos, cosméticos, produtos de higiene, saneamento e outros. Diário Oficial da União 1977; 6 jan.

14. Brasil. Lei no 9.782, de 26 de janeiro de 1999. Define o Sistema Nacional de Vigilância Sanitária, cria a Agência Nacional de Vigilância Sanitária, e dá outras providências. Diário Oficial da União 1999; 27 jan.

15. Deus FJT, Sá PFG. Evolução da normatização de boas práticas de fabricação (BPF) e o seu impacto na qualidade de medicamentos comercializados no Brasil. In: Mostra de Produção Científica da Pós-graduação Lato Sensu da PUC Goiás. http://www.cpgls.pucgoias. edu.br/6mostra/artigos/SAUDE/FERNAN DO\%20JUSTINO\%20TORRES\%20DE\%20 DEUS.pdf (acessado em 15/Mai/2019).

16. Costa EA, Fernandes TM, Pimenta TS. A vigilância sanitária nas políticas de saúde no Brasil e a construção da identidade de seus trabalhadores (1976-1999). Ciênc Saúde Coletiva 2008; 13:995-1004.

17. Agência Nacional de Vigilância Sanitária. Resolução de Diretoria Colegiada no 80, de 18 de março de 2002. Regulamento técnico de registro, alterações e inclusão pós-registro e revalidação dos produtos biológicos. Diário Oficial da União 2002; 19 mar.

18. Agência Nacional de Vigilância Sanitária. Resolução de Diretoria Colegiada no 315, de 26 de outubro de 2005. Regulamento técnico de registro, alterações pós-registro e revalidações dos produtos biológicos terminados. Diário Oficial da União 2005; 27 out.

19. Agência Nacional de Vigilância Sanitária. Resolução de Diretoria Colegiada no 55, de 16 de dezembro de 2010. Dispõe sobre o registro de produtos biológicos novos e produtos biológicos e dá outras providências. Diário Oficial da União 2010; 17 dez.

20. Agência Nacional de Vigilância Sanitária. Resolução de Diretoria Colegiada no 49, de 20 de setembro de 2011. Dispõe sobre a realização de alterações e inclusões pós-registro, suspensão e reativação de fabricação e cancelamentos de registro de produtos biológicos e dá outras providências. Diário Oficial da União 2011; 22 set.

21. BrownI TM, Cueto M, Fee E. A transição de saúde pública "internacional" para "global" e a Organização Mundial da Saúde. Hist Ciênc Saúde-Manguinhos 2006; 13:623-47.

22. Secretaria de Vigilância Sanitária, Ministério da Saúde. Portaria SVS/MS no 16, de 6 de março de 1995. Determina a todos os estabelecimentos produtores de medicamentos, o cumprimento das diretrizes estabelecidas pelo "Guia de Boas Práticas de Fabricação para a Indústria Farmacêutica”. Diário Oficial da União 1995; 7 mar.
23. Agência Nacional de Vigilância Sanitária. Resolução de Diretoria Colegiada no 134, de 13 de julho de 2001. Determina a todos os estabelecimentos fabricantes de medicamentos, o cumprimento das diretrizes estabelecidas no regulamento técnico das boas práticas para a fabricação de medicamentos. Diário Oficial da União 2001; 14 jul.

24. Agência Nacional de Vigilância Sanitária. Resolução de Diretoria Colegiada no 210, de 4 de agosto de 2003. Atualiza as boas práticas de fabricação de medicamentos, com o objetivo de acompanhamento do desenvolvimento de novas tecnologias, nos últimos anos, e a relevância de documentos nacionais e internacionais a respeito do tema. Diário Oficial da União 2003; 14 ago.

25. Agência Nacional de Vigilância Sanitária. Resolução de Diretoria Colegiada no 17, de 16 de abril de 2010. Dispõe sobre as boas práticas de fabricação de medicamentos. Diário Oficial da União 2010; 17 abr.

26. Agência Nacional de Vigilância Sanitária. Resolução de Diretoria Colegiada no 301, de 21 de agosto de 2019. Dispõe sobre as boas práticas de fabricação de medicamentos. Diário Oficial da União 2019; 22 ago.

27. Agência Nacional de Vigilância Sanitária. Instrução Normativa no 45, de 21 de agosto de 2019. Dispõe sobre as boas práticas de fabricação complementares a medicamentos experimentais. Diário Oficial da União 2019; 22 ago.

28. Secretaria de Vigilância Sanitária, Ministério da Saúde. Portaria no 911, de 12 de novembro de 1998. Aprova a relação de documentos necessários à instrução de pedidos de autorização para realização de pesquisa clínica com fármacos, medicamentos, vacinas e testes diagnósticos novos. Diário Oficial da União 1998; 13 nov.

29. Agência Nacional de Vigilância Sanitária. Resolução de Diretoria Colegiada no 219, de 20 de setembro de 2004. Aprova o regulamento para elaboração de dossiê para a obtenção de comunicado especial (CE) para realização de pesquisa clínica com medicamentos e produtos para a saúde. Diário Oficial da União 2004; 21 set.

30. Lanzarini CM, Porto TF, Penido C. Avaliação de ensaios clínicos no Brasil: histórico e atualidades. Rev Bioét 2018; 26:350-9.

31. Agência Nacional de Vigilância Sanitária. Resolução de Diretoria Colegiada no 39, de 5 de junho de 2008. Aprova o regulamento para a realização de pesquisa clínica e dá outras providências. Diário Oficial da União 2008; 6 jun.

32. Agência Nacional de Vigilância Sanitária. Resolução de Diretoria Colegiada no 9, de 20 de fevereiro de 2015. Dispõe sobre o regulamento para a realização de ensaios clínicos com medicamentos no Brasil. Diário Oficial da União 2015; 21 fev. 


\section{Abstract}

The regulation of biological products has evolved rapidly in recent years due to quality issues impacting people's lives and the advent of new technologies, with constant changes in regulations that dictate how a product is registered, produced, and monitored. In the case of vaccines, the responsibility of regulators and manufacturers in guaranteeing quality, safety, and efficacy is even more critical, since vaccines are mostly used in children and healthy patients. In this scenario, manufacturers need to create strategies to keep their products and installations adequate and up-to-date with a fully operational quality system. Meanwhile, regulatory agencies have the role of guaranteeing that products meet the established criteria without compromising the supply of medicines to the population.

Vaccines; Health Authorities; Formal Social Control

\section{Resumen}

La regulación para productos biológicos ha evolucionado rápidamente a lo largo de los últimos años, sea motivada por cuestiones de calidad con impacto en la vida de las personas, o por el advenimiento de nuevas tecnologías. Los cambios en las regulaciones que dictan como un producto debe ser registrado, producido y monitoreado son constantes. La responsabilidad de reguladores y fabricantes en la garantía de la calidad, seguridad y eficacia de las vacunas se convierte en algo todavía más crítico, ya que estas sustancias se utilizan, en su mayoría, en niños y pacientes saludables. Ante este escenario, los fabricantes necesitan crear estrategias para mantener sus productos e instalaciones de forma adecuada, además de un sistema de calidad actualizado y operativo. Por otro lado, las agencias reguladoras tienen el papel de garantizar que los productos que están en uso atiendan a los criterios establecidos, sin comprometer el suministro de medicamentos para la población.

Vacunas; Autoridades de Salud; Control Social Formal
Recebido em 15/Out/2019

Versão final reapresentada em 05/Mar/2020

Aprovado em 20/Mar/2020 